\title{
Simple and Rapid Immobilization of Coating Polymers on Poly(dimethyl siloxane)-glass Hybrid Microchips by a Vacuum- drying Method
}

\author{
Fumihiko Kitagawa, ${ }^{* \dagger}$ Syo Nakagawara,* Isoshi Nukatsuka, * Yusuke Hori, ** \\ Kenji SueYOSHI,*** and Koji OTSUKA** \\ *Department of Frontier Materials Chemistry, Graduate School of Science and Technology, \\ Hirosaki University, 3 Bunkyo-cho, Hirosaki, Aomori 036-8561, Japan \\ **Department of Material Chemistry, Graduate School of Engineering, Kyoto University, Katsura, Nishikyo, \\ Kyoto 615-8510, Japan \\ ***Department of Applied Chemistry, Osaka Prefecture University Graduate School of Engineering, \\ 1-1 Gakuen-cho, Naka, Sakai 599-8531, Japan
}

\begin{abstract}
A simple and rapid vacuum-drying modification method was applied to several neutral and charged polymers to obtain coating layers for controlling electroosmotic flow (EOF) and suppressing sample adsorption on poly(dimethyl siloxane) (PDMS)-glass hybrid microchips. In the vacuum-dried poly(vinylpyrrolidone) coating, the electroosmotic mobility $\left(\mu_{\mathrm{eo}}\right)$ was suppressed from +2.1 to $+0.88 \times 10^{-4} \mathrm{~cm}^{2} / \mathrm{V} \cdot \mathrm{s}$, and the relative standard deviation (RSD) of $\mu_{\mathrm{eo}}$ was improved from 10.2 to $2.5 \%$ relative to the bare microchannel. Among several neutral polymers, poly(vinylalcohol) (PVA) and poly(dimethylacrylamide) coatings gave more suppressed and repeatable EOF with RSDs of less than $2.3 \%$. The vacuum-drying method was also applicable to polyanions and polycations to provide accelerated and inversed EOF, respectively, with acceptable RSDs of less than 4.9\%. In the microchip electrophoresis (MCE) analysis of bovine serum albumin (BSA) in the vacuum-dried and thermally-treated PVA coating channel, an almost symmetric peak of BSA was obtained, while in the native microchannel a significantly skewed peak was observed. The results demonstrated that the vacuum-dried polymer coatings were effective to control the EOF, and reduced the surface adsorption of proteins in MCE.
\end{abstract}

Keywords Microchip electrophoresis, vacuum-drying method, polymer coating, electroosmotic flow, bioanalysis

(Received June 16, 2015; Accepted July 24, 2015; Published November 10, 2015)

\section{Introduction}

In the last quarter-century, microfluidic bioanalytical devices including microchip electrophoresis (MCE), which is based on capillary electrophoresis (CE), have received much attention due to a short analysis time, high-throughput ability, and small sample/ reagent consumptions. ${ }^{1-5}$ In the case of the MCE analysis of biomolecules, irreversible adsorption of the analytes onto the inner surface of the microchannel is often problematic, reducing the separation performances and the analytical reproducibilities. To overcome the drawbacks, various microchannel coating techniques, which are mainly categorized into physically adsorbed and covalently immobilized layer coatings, have been developed to suppress the adsorption of biomolecules in MCE. ${ }^{6-10}$ In the former modification technique, the microchannel is filled with a solution containing modifier materials, such as surfactants and polymers, and then incubated for the immobilization. To facilitate improved binding of coating materials, in some cases, the surface of the microchannel is activated by oxygen plasma and UV irradiation prior to the

$\dagger$ To whom correspondence should be addressed.

E-mail: kitagawa@hirosaki-u.ac.jp coating, or the coating layer is thermally passivated after the channel modification. In the immobilization of charged polymers, a polyelectrolyte multilayers (PEMs) coating technique is generally employed as the alternating adsorption of polyanions and polycations. ${ }^{11}$ A dynamic coating technique, which is carried out by only adding coating polymers into a background solution (BGS), is also useful for surface modification. Although these physical modifications easily give the coating layers, desorption of the coating materials from the channel surface would reduce the analytical reproducibilities.

In our previous study, a poly(vinylalcohol) (PVA) coating onto the poly(dimethyl siloxane) (PDMS) microchannel to suppress both electroosmotic flow (EOF) and sample adsorption was employed for the analysis of oligosaccharides by large-volume sample stacking with an EOF pump (LVSEP) technique. ${ }^{12}$ To prepare the PVA-coated channel, a multi-layer adsorption and heat-immobilization technique, reported by $\mathrm{Wu}$ et al., ${ }^{13}$ was used. Although an EOF-suppressed microchannel was surely obtained, this modification technique had two problems, i.e., a labor-intensive procedure and a low success-yield for the coating. In this coating technique, at first, entire channels were filled with a PVA solution. The chip was incubated for $10 \mathrm{~min}$ at room temperature, then the reservoirs and channels were emptied by a vacuum pump. An adsorbed PVA layer was dried 
by heating at $110^{\circ} \mathrm{C}$ for $15 \mathrm{~min}$. The above procedures were repeated three-times, resulting in a multi-layer PVA coating. Finally, the coating was thermally immobilized at $140^{\circ} \mathrm{C}$ for $20 \mathrm{~min}$. Hence, this multi-layer adsorption technique required three-times solution loading, incubating and drying steps, and ca. $100 \mathrm{~min}$ for completion of the coating. Furthermore, repeated injections of a viscous PVA solution and subsequent thermal-immobilizations sometimes caused clogging of the narrow channel, resulting in a poor success-yield of the coating in our research group. To overcome these drawbacks, we have recently developed a novel vacuum-drying method, ${ }^{14}$ which modified previous approaches reported by Nii ${ }^{15}$ and Sueyoshi, ${ }^{16}$ to immobilize the PVA layer onto the PDMS surface. This coating technique needs only the loading of the PVA solution into the PDMS microchannel which is incubated with a sodium dodecyl sulfate (SDS) solution for several minutes to hydrophilize the PDMS surface, and subsequent vacuum-drying for 5-10 min. However, the technique has been applied only to PVA, chitosan and methyl cellulose,${ }^{14}$ and detailed comparisons of the EOF properties on the prepared coating layers have not been made. To widen the applicability of our developed method, in this study, several neutral and charged polymers were immobilized via vacuum-drying to obtain coating layers for controlling EOF and suppressing sample adsorption on PDMSglass hybrid microchips. The stabilities of the EOF in the modified channels were investigated. In addition, the coating channel obtained by a vacuum-drying method was applied to the MCE-LVSEP analysis of bovine serum albumin to verify the effectiveness of the method for reducing the surface adsorption of proteins.

\section{Experimental}

\section{Chemicals}

Poly(vinylpyrrolidone) (PVP, $\left.M_{\mathrm{W}} 360000\right)$, PVA ( $M_{\mathrm{W}} 85000-$ $124000,87-89 \%$ hydrolyzed), hydroxyethyl cellulose (HEC, $M_{\mathrm{W}}$ 250000), dextran sulfate (DxS, $\left.M_{\mathrm{W}}>500000\right)$, poly (ethylenimine) (PEI, $M_{\mathrm{W}} \sim 750000$ ), poly(dimethylacrylamide) (PDMA, $M_{\mathrm{n}}$ 10000), and fluorescein isothiocyanate (FITC)-labeled bovine serum albumin (BSA) were obtained from Sigma-Aldrich (Tokyo, Japan). PDMS base and curing reagent (Sylgard 184) were from Dow Corning Toray (Tokyo, Japan) and SU8-50 from MicroChem (Newton, MA). SDS and all other reagents were purchased from Wako (Osaka, Japan). All reagents were of analytical or HPLC grade. Background solutions (BGSs) used throughout the experiments were $25-50 \mathrm{mM}$ phosphate buffer (pH 3.1, 7.0). For the LVSEP experiments, FITC-BSA was dissolved in deionized water to yield standard solutions of $1000 \mathrm{ppm}$. Sample solutions $(50-200 \mathrm{ppm})$ were prepared by dilution of the standard solutions with deionized water. All solutions were prepared with deionized water purified by AutoStill (WG510, Yamato Scientific, Tokyo, Japan) and SimplicityUV (Merck Millipore, Billerica, MA) systems, and filtered through a $0.45 \mu \mathrm{m}$ pore membrane filter prior to use.

\section{Procedures}

A PDMS microchannel was fabricated by the conventional procedure based on a soft-lithography using an SU-8 mold and PDMS. Patterned PDMS microchannel plates consisting of a single straight channel $(100 \mu \mathrm{m}$ width $\times 100 \mu \mathrm{m}$ depth $)$ with a total separation channel length of $60.0 \mathrm{~mm}$ were directly compressed onto slide-glass lids $(76.2 \times 25.4 \mathrm{~mm})$, producing PDMS-glass hybrid microchips. For coating the inner surface of the microchannel with several modification-polymers, the vacuum-drying method was applied. ${ }^{14}$ Briefly, a $10 \mathrm{mM}$ SDS solution was manually introduced into the fabricated microchannel using a micro-syringe to hydrophilize the PDMS surface. After removing the SDS solution, the modification polymer solution was injected into the microchannel. The microchip was put into a vacuum-chamber under reducedpressure $(<\sim 1$ Torr) by using an oil rotary vacuum pump (G-50DA, ULVAC KIKO, Saito, Japan) for $10 \mathrm{~min}$. The coated channel was washed with water, and finally conditioned with the BGS.

\section{MCE experiments}

To evaluate the microchannel coating, the electroosmotic mobility $\left(\mu_{\mathrm{eo}}\right)$ was measured by the current-monitoring technique. ${ }^{13,17-19}$ All channels were first filled with a $50 \mathrm{mM}$ phosphate buffer, and a $25 \mathrm{mM}$ phosphate buffer was injected into the cathodic buffer port. Then, a potential of $1.00 \mathrm{kV}$ $(167 \mathrm{~V} / \mathrm{cm})$ was applied by a high-voltage sequencer (HVS4486000D-ST, LabSmith, CA) across the whole straight channel, and the current through the channel was monitored. From the current-monitoring, the time required for replacing the $25 \mathrm{mM}$ buffer was measured to determine $\mu_{\mathrm{e}}$.

MCE experiments were performed on a fluorescence microscope (IX73, Olympus, Tokyo, Japan). A $130 \mathrm{~W}$ mercury lamp (SHI-1300L, Olympus) for excitation was introduced to the microscope, and irradiated to the microchannel through a dichroic filter (BP460-480GFP, Olympus) and an objective lens (LUCPLFLN 20×, Olympus). Fluorescence from analytes collected by the same objective lens was passed through a dichroic filter (U-FBWA, Olympus), and led to a multichannel photodetector (PMA-12, Hamamatsu Photonics, Hamamatsu, Japan). In the MCE-LVSEP analysis, a sample solution was introduced into the entire channel by using a syringe manually. The two reservoirs were filled with $3 \mu \mathrm{L}$ of $25 \mathrm{mM}$ phosphate buffer ( $\mathrm{pH} 7.0$ ). A voltage of $-1.0 \mathrm{kV}$ was applied through two platinum electrodes immersed in the two reservoirs. The analytes were detected by the fluorescence detection scheme at the point $5.0 \mathrm{~mm}$ from the anodic end.

\section{Results and Discussion}

\section{Comparison with dynamic coating}

In our previous study, the vacuum-drying PVA coating was applied to PDMS microchips bonded with $\mathrm{O}_{2}$ plasma irradiation, resulting in stable EOF during 30 runs. $^{14}$ Since the PDMS channel plate and glass lid were gradually detached, the PDMSglass hybrid microchips could not be used for over 20 runs. In this study, hence, the EOF stability was mainly evaluated within consecutive 10 runs. First, to evaluate the effectiveness of the vacuum-drying method, the electroosmotic mobilities obtained in bare PDMS-glass hybrid (no coating), dynamic and vacuumdrying coating channels were compared. For this purpose, PVP was selected as the coating polymer, since it is widely used for the dynamic coating in both $\mathrm{CE}^{20}$ and MCE. ${ }^{21}$ Also, a permanent PVP coating onto the PDMS channel was reported. ${ }^{22}$ Figure 1 shows the changes in the $\mu_{\mathrm{eo}}$ in the bare, dynamic and vacuumdrying PVP coating channels. Apparently, the EOF in the bare channel was unstable, while dynamic and vacuum-drying coatings gave more suppressed and stable EOFs. As summarized in Table $1, \mu_{\text {ео }}$ in the vacuum-dried PVP coating channel was almost identical with that in the dynamic PVP coating, +8.7 $8.8 \times 10^{-5} \mathrm{~cm}^{2} / \mathrm{V} \cdot \mathrm{s}$. It should be noted that the relative standard deviation (RSD) of $\mu_{\mathrm{eo}}$ obtained with the vacuum-drying method was improved from 7.0 to $2.5 \%$ compared to the dynamic 


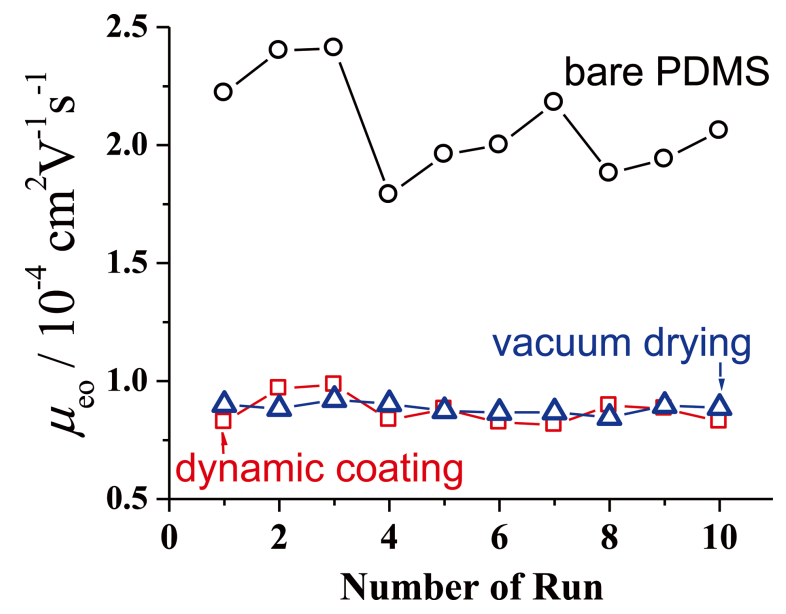

Fig. 1 EOF stability in the bare PDMS-glass hybrid (open circles), dynamically PVP-coated (open squares) and PVP-coated channels using the vacuum-drying method (open triangles). PVP concentration, $0.1 \%$; BGS, $50 \mathrm{mM}$ phosphate buffer (pH 7.0).

Table $1 \mu_{\mathrm{eo}}$ in the PVP-coated channels ${ }^{\mathrm{a}}$

\begin{tabular}{lcc}
\hline \multicolumn{1}{c}{ Coating } & $\mu_{\mathrm{eo}}\left(\mathrm{cm}^{2} / \mathrm{V} \cdot \mathrm{s}\right)$ & $\% \mathrm{RSD}(n=10)$ \\
\hline Bare (no coating) & $+2.1 \times 10^{-4}$ & 10.2 \\
Dynamic PVP coating & $+8.7 \times 10^{-5}$ & 7.0 \\
PVP coating by vacuum-drying & $+8.8 \times 10^{-5}$ & 2.5 \\
\hline
\end{tabular}

a. PVP concentration, $0.1 \%$; BGS, $50 \mathrm{mM}$ phosphate buffer (pH 7.0).

coating. In the dynamic coating technique, generally, higher polymer contents give more suppressed and repeatable $\mu_{\mathrm{eo}}$, whereas longer migration times are sometimes problematic due to the increased viscosity. On the other hand, the vacuumdrying PVP coating resulted in better repeatability without any conditioning between the runs and recoating the polymers. Thus, the vacuum-drying method is effective to obtain stable EOF with simple and rapid procedures.

\section{Application to neutral coating-polymers}

In the MCE analysis of biomolecules, EOF is often suppressed by the coating polymers, which also block the sample adsorption onto the inner surface of the microchannel. To evaluate the applicability of the vacuum-drying method, we selected four neutral coating polymers, i.e., PVP, PVA, HEC and PDMA, which are extensively employed in the CE and MCE analysis of biomolecules. ${ }^{910}$ Figure 2 shows the EOF stability in these polymers coated microchannels using the vacuum-drying method and the results are summarized in Table 2. By comparing with the PVP coating, PVA and PDMA polymers gave slightly suppressed and stable EOF with the RSDs less than $2.3 \%$, while in the HEC-coated channel less repeatable EOF (RSD of $4.1 \%$ ) was observed. Considering that the hydroxypropyl methylcellulose (HPMC, $M_{\mathrm{W}}$ 22000)-coated channel also resulted in poor repeatability (data not shown), cellulose polymers may not be well immobilized onto the inner surface of the PDMS-glass hybrid microchips by the vacuumdrying method. It should be emphasized that relatively viscous polymers including PVA and HEC could be modified with acceptable EOF performances without any channel clogging, which is sometimes problematic in the polymer-coating of the narrow microchannel. Such successful coatings of neutral polymers are useful for analyzing biomolecules in MCE.

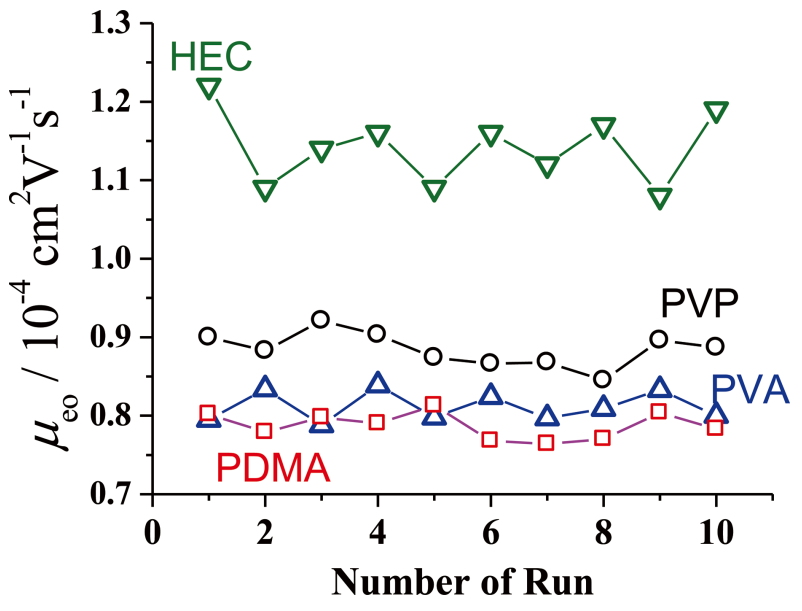

Fig. 2 EOF stability in the neutral polymer coating microchannels using the vacuum-drying method. Open circles, PVP; triangles, PVA; squares, PDMA; inverted triangles, HEC. Polymer concentrations, $0.1 \%$; BGS, $50 \mathrm{mM}$ phosphate buffer ( $\mathrm{pH} 7.0)$.

Table $2 \mu_{\text {eo }}$ in the neutral polymer coating channels using the vacuum-drying method ${ }^{\mathrm{a}}$

\begin{tabular}{lcc}
\hline Coating & $\mu_{\mathrm{eo}}\left(\mathrm{cm}^{2} / \mathrm{V} \cdot \mathrm{s}\right)$ & $\% \mathrm{RSD}(n=10)$ \\
\hline PVP & $+8.8 \times 10^{-5}$ & 2.5 \\
PVA & $+8.1 \times 10^{-5}$ & 2.3 \\
PDMA & $+7.9 \times 10^{-5}$ & 2.2 \\
HEC & $+1.1 \times 10^{-4}$ & 4.1 \\
\hline
\end{tabular}

a. Polymer concentrations, $0.1 \%$; BGS, $50 \mathrm{mM}$ phosphate buffer (pH 7.0).

The effect of the vacuum-drying time on the coating stability was studied. In the case of the vacuum-drying of PVA, significant changes in $\mu_{\mathrm{eo}}$ and the RSDs were not observed, $+8.0-8.1 \times 10^{-5} \mathrm{~cm}^{2} / \mathrm{V} \cdot \mathrm{s}$ and $2.3-2.5 \%$, respectively, at drying times of 10, 30 and $60 \mathrm{~min}$. Thus, the vacuum-drying time of 10 min was selected in the remaining study. As for the PVA coating, a thermal treatment of the polymer layer after vacuumdrying is expected to provide more stable EOF and superior separation performances due to suppressing the sample adsorption. Since thermally passivated PVA film contains polymer brushes on the surface, the brush structure causes strong steric repulsive forces between the PVA surface and analyte molecules. ${ }^{23-25}$ The effect of the thermal treatment after vacuum-drying on the EOF stability in the PVA-coated microchannels was investigated. After heating at $110^{\circ} \mathrm{C}$ for $60 \mathrm{~min}$, an apparent decrease in $\mu_{\mathrm{eo}}$ from +8.1 to $+7.0 \times$ $10^{-5} \mathrm{~cm}^{2} / \mathrm{V} \cdot \mathrm{s}$ was observed, as shown in Fig. 3. In the thermallypassivated PVA channel, furthermore, $\mu_{\mathrm{eo}}$ was decreased slightly around the first four runs, followed by an almost constant EOF velocity to the 14th run. This would be due to insufficient conditioning of the PVA passivated channel, i.e., long surface equilibration with the BGS may be needed after the thermal treatment. The RSD value of $\mu_{\mathrm{eo}}$ in the prepared coating channel was calculated to be $1.1 \%$ from fifth to fourteenth runs, which was better than the PVA coated channel without any heating treatment (RSD of 2.3\%). The vacuum-dried and thermallypassivated PVA coating channel showed superior EOF properties, and was thus applied to the MCE analysis of proteins in the following section. 


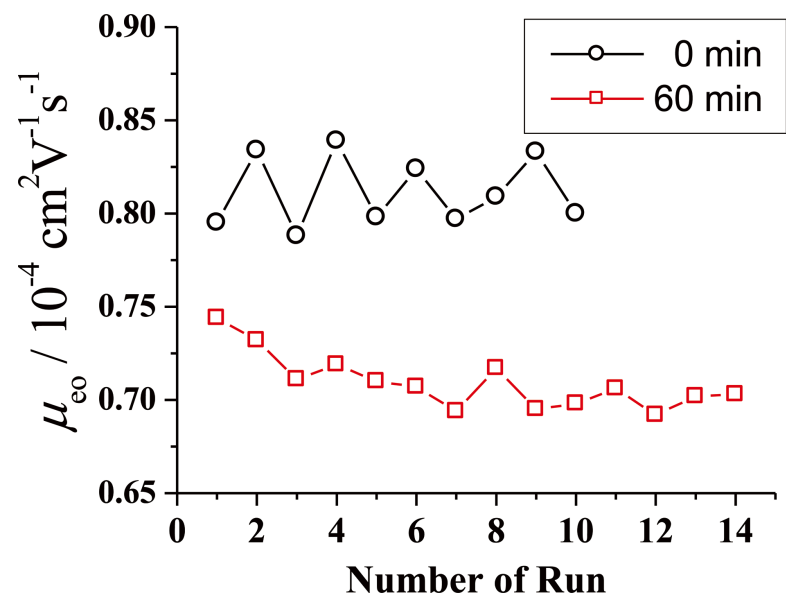

Fig. 3 Effect of a heating treatment on the EOF stability in the PVAcoated microchannels using a vacuum-drying method. PVA concentration, $0.1 \%$; BGS, $50 \mathrm{mM}$ phosphate buffer ( $\mathrm{pH} 7.0$ ); heating temperature, $110^{\circ} \mathrm{C}$.

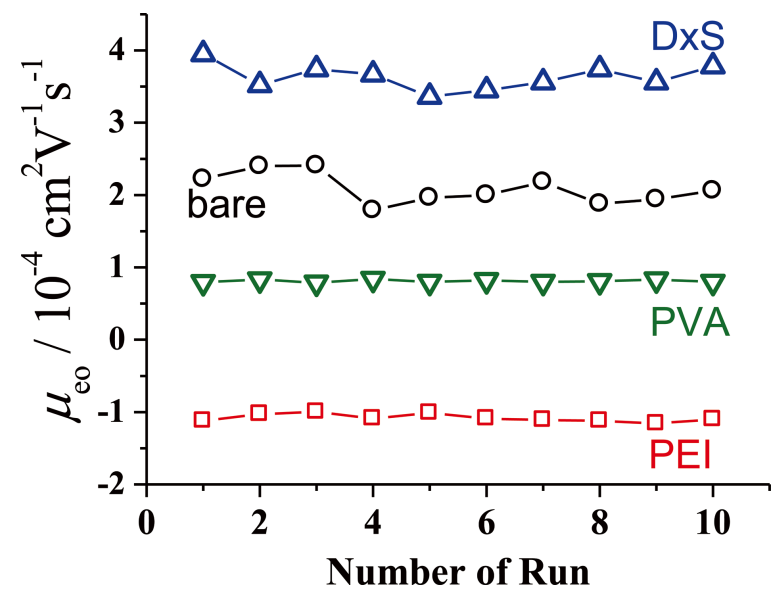

Fig. 4 EOF stability in the charged polymer coating channels using the vacuum-drying method. Polymer concentrations, 5.0\%; BGS, $50 \mathrm{mM}$ phosphate buffer at $\mathrm{pH} 7.0$ for bare, PVA and DxS, and at $\mathrm{pH} 3.1$ for PEI.

\section{Application to charged coating-polymers}

Charged polymers are used as adsorbed coatings to obtain accelerated or reversed EOF in the microchannel. Since the glass and PDMS channel surfaces are often negatively-charged, cationic polymers including polybrene, chitosan, poly(diallyldimethylammonium chloride) and PEI are statically or dynamically immobilized onto these inner walls as the first layer, resulting in reversed EOF. ${ }^{6,16}$ When anionic polymers are modified as the second layer, on the other hand, fast and cathodic EOF occurs.

In applications of the vacuum-drying method to coating charged polymers, PEI and DxS were chosen for accelerating and inverting the EOF, respectively. Figure 4 and Table 3 demonstrate the EOF stability in DxS and PEI coated microchannels. In the DxS coated microchannel, a faster EOF $\left(+3.6 \times 10^{-4} \mathrm{~cm}^{2} / \mathrm{V} \cdot \mathrm{s}\right)$ was obtained relative to the bare channel $\left(+2.1 \times 10^{-4} \mathrm{~cm}^{2} / \mathrm{V} \cdot \mathrm{s}\right)$. The repeatability of the EOF was improved from 10.2 to $4.8 \%$ by the vacuum-drying of DxS. It should be noted that no degradation of the EOF was observed until the tenth run. Although the glass substrate was negatively
Table $3 \mu_{\mathrm{eo}}$ in the charged polymer coating channels using the vacuum-drying method ${ }^{\mathrm{a}}$

\begin{tabular}{ccc}
\hline Coating & $\mu_{\text {eо }}\left(\mathrm{cm}^{2} / \mathrm{V} \cdot \mathrm{s}\right)$ & $\% \operatorname{RSD}(n=10)$ \\
\hline DxS $^{\mathrm{b}}$ & $+3.6 \times 10^{-4}$ & 4.8 \\
PEI $^{\mathrm{c}}$ & $-1.1 \times 10^{-4}$ & 4.9 \\
\hline
\end{tabular}

a. Polymer concentrations, $5.0 \%$.

b. BGS, $50 \mathrm{mM}$ phosphate buffer (pH 7.0).

c. BGS, $50 \mathrm{mM}$ phosphate buffer ( $\mathrm{pH} 3.1)$.

charged, anionic DxS was stably immobilized on the channel surface. This may be due to forced-attachment of DxS onto the channel surfaces via the layer formation under the vacuumdrying condition. This result clearly demonstrated the effectiveness of the vacuum-drying method. Compared to the PEMs coating technique, which usually needs multiple coating steps to immobilize anionic polymers, furthermore, only one modification step is required without any surface regeneration in our proposed method.

As shown in Fig. 4, on the other hand, a stable and reversed EOF with $-1.1 \times 10^{-4} \mathrm{~cm}^{2} / \mathrm{V} \cdot \mathrm{s}$ at $\mathrm{pH} 3.1$ was observed in the PEI-coated channel. At $\mathrm{pH} 7.0$, unfortunately, $\mu_{\text {eo }}$ could not be determined due to a too long time of the current recovery, suggesting a slower EOF due to nearly zero net-charge on the PEI coating surface. This may be caused by a less amount of the immobilized PEI onto the channel surface compared to previous polycations-coated columns. ${ }^{26,27}$ An increased amount of immobilized PEI in the vacuum-dried coating would be attained by multiple deposition of PEI to obtain faster reversedEOF. The run-to-run RSD of the EOF velocity in the PEI coating was estimated to be $4.9 \%$, which was comparable to that of the DxS coated channel. These results clearly indicated that the vacuum-drying technique can be applied to the formation of positively- and negatively-charged coating layers for controlling the EOF.

\section{Application to MCE analysis of proteins}

BSA was analyzed in the vacuum-dried PVA coating channel with the thermal treatment at $110^{\circ} \mathrm{C}$ for 60 min to verify the applicability to the MCE analysis of biomolecules. Since the straight channel was employed in this study, the LVSEP technique, which can enrich and separate the analytes introduced into the entire channel, was applied. ${ }^{12}$ Figure 5 shows the MCELVSEP analysis of BSA in the bare and PVA coated microchannels at pH 7.0. As shown in Fig. 5(a), BSA concentrated by the LVSEP effect was detected as a single peak with a significant peak-leading in the bare microchannel. Since the migration direction of the analytes is inversed between the enrichment and separation steps, the effect of irreversible adsorption or slow retention kinetics of solutes onto the channel surface are observed as the peak-leading in the LVSEP technique. ${ }^{12,28}$ As expected, the effect of the surface adsorption of the analyte proteins was not negligible on the native PDMSglass hybrid microchips. On the other hand, the peak-leading did not appear in the PVA coated channel (Fig. 5(b)), demonstrating effective suppression of the sample adsorption.

To evaluate the effect of the surface adsorption on the MCE analytical performance, the asymmetry factor $\left(A_{\mathrm{S}}\right)$ was calculated. The value of $A_{\mathrm{S}}$ is given by the ratio of $w_{1}$ and $w_{2}$, where $w_{1}$ and $w_{2}$ are the widths measured at a given fraction at $10 \%$ of the maximum peak height before and after the time of peak maximum, respectively. The $A_{\mathrm{S}}$ value obtained in the native microchannel was 0.51 , while in the PVA coated channel 


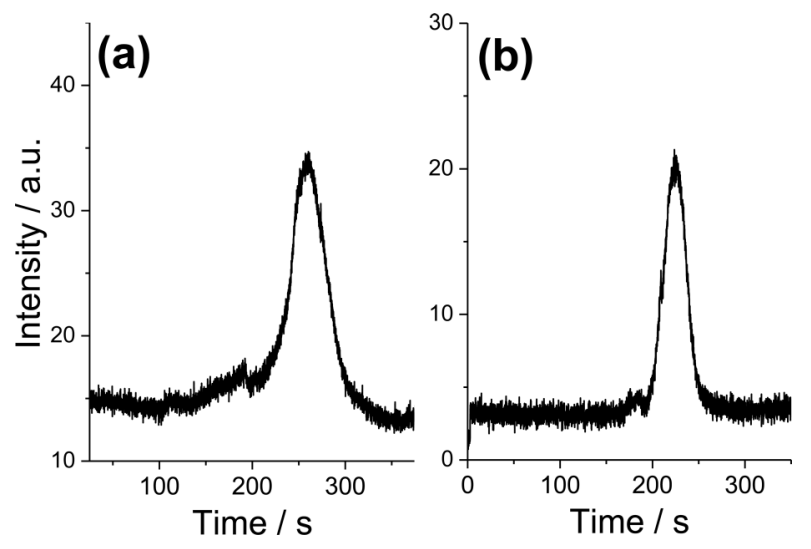

Fig. 5 MCE-LVSEP analyses of BSA obtained with the (a) bare and (b) PVA-coated channel using the vacuum-drying method with the thermal treatment at $110^{\circ} \mathrm{C}$ for $60 \mathrm{~min}$. BGS, $25 \mathrm{mM}$ phosphate buffer (pH 7.0); sample concentrations, (a) 200 and (b) $50 \mathrm{ppm}$. Other conditions are as in the Experimental section.

$A_{\mathrm{S}}$ was almost unity (0.96). Hence, the irreversible adsorption of BSA was successfully suppressed by the PVA layer formed by the vacuum-drying and thermal treatments. Furthermore, the RSD for the migration time of BSA was determined to be $3.8 \%$ $(n=3)$, which was comparable with that in the conventional microchip zone electrophoretic analysis. ${ }^{12}$ Considering the improved peak profile and acceptable repeatability, therefore, the vacuum-drying modification of the coating polymers should be effective to provide high-performance separation media for biomolecules. Unfortunately, the width of the BSA peak obtained in this study was still broader due to the poor stacking efficiency for proteins in the LVSEP experiments. Thus, protein mixtures are now trying to be separated by conventional zone electrophoresis on PVA-coated cross-channel chips.

\section{Conclusions}

On PDMS-glass hybrid microchips, simple and rapid immobilization of both neutral and charged polymers onto the inner surface of the microchannel was successfully achieved by the vacuum-drying method. In the vacuum-dried coating of all tested six polymers (PVP, PVA, HEC, PDMA, DxS and PEI), the drying-time was within $10 \mathrm{~min}$, and no clogging of the channel was found. By coating neutral polymers including PVP, PVA, PDMA and HEC, EOF-suppressed channels were easily obtained with acceptable repeatabilities. The immobilization of DxS and PEI gave accelerated and inversed EOF, respectively. In the vacuum-dried and thermally-treated PVA coating channel, MCE-LVSEP analyses of BSA gave approximately Gaussian peaks, demonstrating the reduction of irreversible adsorption of proteins. We believe that our modification technique is also applicable to most coating polymers to attain highly efficient analyses of a wide variety of biomolecules. However, further suppression or acceleration of the EOF with better repeatabilities are desired to achieve faster and reproducible separation in MCE. Furthermore, the obtained BSA peak was still broader. Therefore, the use of a higher polymer concentration in the vacuum-drying step, multiple coatings, the combination of the thermal treatment, and so on, should be investigated to realize such superior coatings. Work along this line is now progressing in this laboratory.

\section{Acknowledgements}

This work was supported in part by the Grant-in-Aid for Scientific Research (C) (Nos. 24550090 and 15K05527) from the Japan Society for the Promotion of Science (JSPS). This research was also supported by SENTAN, JST.

\section{References}

1. E. R. Castro and A. Manz, J. Chromatogr. A, 2015, 1382, 66.

2. N. Nuchtavorna, W. Suntornsukc, S. M. Lunted, and L. Suntornsuk, J. Pharm. Biomed. Anal., in press, doi:10.1016/ j.jpba.2015.03.002.

3. M. C. Breadmore, J. Chromatogr. A, 2012, 1221, 42.

4. V. Dolnik and S. Liu, J. Sep. Sci., 2005, 28, 1994.

5. V. Dolnik, S. Liu, and S. Jovanovich, Electrophoresis, 2000, $21,41$.

6. J. Zhou, A. V. E. Ellis, and N. H. Voelckerm, Electrophoresis, 2010, 31, 2.

7. J. Liu and M. L. Lee, Electrophoresis, 2006, 27, 3533.

8. D. Belder and M. Ludwig, Electrophoresis, 2003, 24, 3595.

9. E. A. S. Doherty, R. J. Meagher, M. N. Albarghouthi, and A. E. Barron, Electrophoresis, 2003, 24, 34.

10. J. Horvath and V. Dolnik, Electrophoresis, 2001, 22, 644.

11. S. L. R. Barker, M. J. Tarlov, H. Canavan, J. J. Hickman, and L. E. Locascio, Anal. Chem., 2000, 72, 4899.

12. T. Kawai, K. Sueyoshi, F. Kitagawa, and K. Otsuka, Anal. Chem., 2010, 82, 6504.

13. D. Wu, Y. Luo, X. Zhou, Z. Dai, and B. Lin, Electrophoresis, 2005, 26, 211.

14. K. Otsuka, K. Sueyoshi, Y. Hori, and F. Kitagawa, Japanese Patent Application, 2014, 069677.

15. K. Nii, K. Sueyoshi, K. Otsuka, and M. Takai, Microfluid. Nanofluid., 2013, 14, 951.

16. K. Sueyoshi, Y. Hori, and K. Otsuka, Microfluid. Nanofluid., 2013, 14, 933.

17. X. H. Huang, M. H. Gordon, and R. N. Zare, Anal. Chem., 1988, 60, 1837.

18. J. L. Pittman, C. S. Henry, and S. D. Gilman, Anal. Chem., 2003, 75, 361.

19. I. Rodríguez and N. Chandrasekhar, Electrophoresis, 2005, 26, 1114.

20. J. Horvath and V. Dolnik, Electrophoresis, 2001, 22, 644.

21. J. A. Kim, J. Y. Lee, S. Seong, S. H. Cha, S. H. Lee, J. J. Kim, and T. H. Park, Biochem. Eng. J., 2006, 29, 91.

22. D. P. Wu, B. X. Zhao, Z. P. Dai, J. H. Qin, and B. C. Lin, Lab Chip, 2006, 6, 942.

23. M. Gilges, M. H. Kleemiss, and G. Schomburg, Anal. Chem., 1994, 66, 2038.

24. Y. Okamoto, F. Kitagawa, and K. Otsuka, Electrophoresis, 2006, 27, 1031.

25. Y. Ikada, H. Iwata, F. Horii, T. Matsunaga, M. Taniguchi, M. Suzuki, W. Taki, S. Yamagata, Y. Yonekawa, and H. Handa, Biomed. Mater. Res., 1981, 15, 697.

26. F. B. Erim, A. Cifuentes, H. Poppe, and J. C. Kraak, J. Chromatogr. A, 1995, 708, 356.

27. F. Kitagawa, M. Kamiya, Y. Okamoto, H. Taji, S. Onoue, Y. Tsuda, and K. Otsuka, Anal. Bioanal. Chem., 2006, 386, 594.

28. F. Kitagawa, T. Kawai, and K. Otsuka, Anal. Sci., 2013, 29, 1129. 